\title{
Desarrollo del proceso de modelación matemática en licenciados en formación*
}

\author{
Jesús David Berrío Valbuena ${ }^{1}$ \\ Zuriel Fitzgerald Peña Ubarne ${ }^{2}$ \\ María de los Ángeles Torrenegra Giraldo ${ }^{3}$
}

Recibido: $11-10-2019$

Aceptado: 31-10-2019

\section{Resumen}

Para autores como Blum, Galbraith, Henn y Niss (2007), la modelación tiene su punto de partida en la conceptualización de una situación o problema de la realidad. Para Blum y BorromeoFerri (2009), este proceso se ve esquematizado en un ciclo que tiene ciertas fases que permiten un razonamiento adecuado de los problemas de modelación. En este artículo se propone un ciclo basado en dichos autores y en el contexto estudiado de los licenciados en formación de la Universidad del Atlántico, luego de lo cual se realiza un contraste entre el ciclo propuesto y los procesos que desarrollan los estudiantes cuando resuelven un problema de modelación matemática planteado desde la experimentación.

\footnotetext{
* Este texto expone los resultados de una investigación en curso llevada a cabo en la Universidad del Atlántico. El tipo de texto es artículo corto.

1. Licenciado en Matemáticas de la Universidad del Atlántico y magíster en Educación Matemática de la Universidad Industrial de Santander. Docente investigador del programa de Licenciatura en Matemáticas, Universidad del Atlántico, Barranquilla, Colombia.

Correo electrónico: iberriovalbuena@mail.uniatlantico.edu.co

ORCID: https://orcid.org/0000-0002-4014-5322

Google académico: https://scholar.google.es/citations?user=088Z5lkAAAAJ\&hl=es
}

2. Estudiante de Licenciatura en Matemáticas de la Universidad del Atlántico, Barranquilla, Colombia.

Correo electrónico: zpena@mail.uniatlantico.edu.co

ORCID: https://orcid.org/0000-0001-8130-5794

Google académico: https://scholar.google.com/citations?user=ttriapoAAAAJ\&hl=es

3. Estudiante de Licenciatura en Matemáticas de la Universidad del Atlántico. Barranquilla - Colombia.

Correo electrónico: mdtorrenegra@mail.uniatlantico.edu.co

ORCID: https://orcid.org/0000-0001-6395-7692

Google académico: https://scholar.google.com/citations?user=TOc9Cl gAAAAJ\&hl=es 
Palabras clave: modelo matemático, ciclo de modelación, experimentación, contexto.

\section{Development of the mathematical modeling process in training graduates}

\section{Abstract}

For authors such as Blum, Galbraith, Henn and Niss (2007), modeling has its starting point in the conceptualization of a situation or problem of reality. For Blum and Borromeo-Ferri (2009), this process is schematized in a cycle that has certain phases that allow an adequate reasoning of the modeling problems. This article proposes a cycle based on these authors and in the studied context of the graduates in training of the Universidad del Atlántico, after which a contrast is made between the proposed cycle and the processes that students develop when they solve a problem mathematical modeling raised from experimentation.

Keywords: mathematical model, modeling cycle, experimentation, context.

\section{Introducción}

La necesidad de describir y estudiar la realidad constituye un aspecto importante en el desarrollo humano, ante los distintos avances tecnológicos y económicos se requiere de individuos que relacionen las matemáticas con su entorno. De ahí que la modelación matemática sea considerada una alternativa pertinente, pues permite desarrollar habilidades para comunicarse matemáticamente, expresar ideas, describir relaciones propias del entorno y además 
representar y evaluar situaciones cotidianas (Ministerio de Educación Nacional [MEN], 1998).

Por tanto, se hace necesario que los docentes reconozcan la modelación matemática como un recurso en el aula de clases que permite relacionar las matemáticas con situaciones de la vida cotidiana (Pantoja, Guerrero, Ulloa y Valdivia, 2016), mediante la elaboración e interpretación de modelos que le den significado a los conceptos matemáticos para así despertar interés y motivación en los estudiantes, puesto que ellos pueden interactuar con el contexto a partir del planteamiento de una situación problema (Villa-Ochoa y Ruiz, 2009). Con el desarrollo de tales habilidades se espera que el alumno pueda articular las matemáticas con otras áreas del conocimiento, además de mejorar su capacidad de leer, interpretar, formular y resolver situaciones problema, entre otros aspectos (Biembengut y Hein, 2004).

Según Trigueros (2009), la modelación matemática en la escuela se muestra de diferentes maneras; una de ellas se denomina modelación educativa, y tiene un objetivo pedagógico que distingue dos tipos de corrientes: una didáctica, en la cual el modelo desarrollado permite la promoción y estructuración de los procesos de aprendizaje de los estudiantes; y otra en la que la modelación es importante para la adquisición y el desarrollo de nuevos conceptos.

En desarrollo de lo anterior, para la implementación de la modelación en el aula algunos autores como: Villa-Ochoa y Ruiz (2009), Rodríguez (citado por Rodríguez, 2010), y Blum y Borromeo-Ferri (2009) han determinado un ciclo de modelación, el cual subraya la forma como este proceso se desarrolla. Esto le permite al profesor la planificación de actividades y estrategias para el desarrollo de situaciones experimentales llevadas al aula y, de esta manera, tener éxito en su aplicación, pues la implementación de la modelación en el aula ha demostrado el desarrollo de competencias matemáticas, así como la promoción del interés hacia la asignatura y la formación de un pensamiento diversificado en los estudiantes (Rodríguez y Quiroz, 2016). 
En el presente artículo se busca mediante la implementación de una actividad propuesta describir el proceso de modelación que surge del desarrollo de esta con los estudiantes de la asignatura Didáctica del Cálculo del programa de Licenciatura en Matemáticas de la Universidad del Atlántico; de tal manera que se pueda esquematizar dicho proceso con el fin de contrastarlo con el ciclo de modelación desarrollado por los investigadores a partir del propuesto por Borromeo-Ferri (citado por Huincahue, Borromeo-Ferri y Mena-Lorca, 2018).

Para esto se introduce el modelo de elaboración propia, y mediante un análisis a priori se describen los pasos que los licenciados en formación deben seguir para el desarrollo de la actividad; luego mediante un análisis a posteriori se describen los procesos que los licenciados en formación siguieron en la resolución de la actividad, lo que genera un esquema general de este proceso y al final se contrasta con el ciclo de elaboración propia.

\section{Modelación matemática}

Desde la perspectiva de Blum, Galbraith, Henn y Niss (2007), la modelación puede considerarse como un proceso que tiene su génesis en la conceptualización de una situación o problema de la realidad. Para Camarena (citado por Morales y Currea, 2019), "la modelación matemática se concibe como el proceso cognitivo que se tiene que llevar a cabo para llegar a la construcción del modelo matemático de un evento u objeto del área del contexto" (p. 18). En este sentido, Villa- Ochoa, Bustamante y Berrío (2010) argumentan que dicho proceso tiene "como punto de partida un conjunto de situaciones asociadas a los diferentes contextos sociales y culturales de los estudiantes y la escuela" (p. 1089), opinión que concuerda con lo establecido por el MEN (1998), cuando afirma que "es necesario relacionar los contenidos de aprendizaje con la experiencia cotidiana de los alumnos, así como presentarlos y enseñarlos en un contexto de situaciones problemáticas de intercambio de puntos de vista" (p. 18). Autores como Ortega, Zamora, 
Ulloa y González (2018) reafirman que la modelación matemática, al relacionar el saber matemático con la vida cotidiana, favorece la generación de competencias para el trabajo colaborativo, la elaboración de reportes, la discusión de los resultados ante el grupo y la interpretación de las diferentes representaciones semióticas en función de la situación problema.

Entre tanto, autores como Suárez y Cordero (2010) adoptan la modelación

como una construcción teórica que un individuo realiza al enfrentar una tarea matemática en la que pone en juego sus conocimientos. Y como características propias de esta construcción la modelación posee su propia estructura, está constituida por un sistema dinámico, la simulación puede llevar a cabo realizaciones múltiples y hacer ajustes en su estructura para producir un resultado deseable, es un medio que propicia el desarrollo del razonamiento y de la argumentación, busca explicaciones a un rango y enfatiza invariantes, trae una idea en una realización para satisfacer un conjunto de condiciones. (pp. 320-321)

A partir de esto, entendemos por modelación matemática el proceso constituido por una serie de fases o acciones denominadas ciclos de modelación, el cual inicia con la determinación de un problema o un fenómeno de la realidad, que posteriormente es sometido a la observación y a la experimentación con el fin de obtener datos que permitan comprender o identificar a profundidad todos los actores involucrados en dicho fenómeno, con el objetivo de determinar la construcción más adecuada que represente el fenómeno estudiado (Villa-Ochoa y Ruiz, 2009). Así, la modelación matemática, al describir un proceso que involucra al mundo real y las matemáticas, constituye una herramienta didáctica que permite la construcción del conocimiento matemático (Berrío, Peña y Torrenegra, 2018), pues si en el aula de clase la enseñanza de un objeto matemático no se desarrolla a través de la modelación o su relación con situaciones de la realidad ocasiona la des-significación del objeto como elemento cognitivo, y además se trata al modelo 
como un objeto del intelecto, es decir, en la mera abstracción y no visto como un proceso (Fernández-Sánchez y Angulo-Cruz, 2019).

Por otra parte, para Biembengut y Hein (1999), la modelación matemática o modelaje matemático es un proceso que

puede ser considerado artístico, ya que, para elaborar un modelo, además del conocimiento profundo de matemáticas, el modelador debe tener una dosis de intuicióncreatividad, para interpretar el contexto, discernir qué contenido matemático se adapta mejor y sentido lúdico para jugar con las variables involucradas [...]. Las expresiones creadas deberán servir no solo para una solución particular, sino posteriormente, como soporte para otras aplicaciones y teorías. (p. 120-121)

Luego, la modelación matemática favorece la resolución de problemas y el desarrollo de la actividad científica en el aula de clases, pues permite relacionar las matemáticas con otras áreas del saber $y$, además, los modelos planteados no solo pueden explicar un fenómeno único, sino que también pueden servir para explicar otras situaciones diferentes en otros contextos.

Como ya se ha indicado, este estudio se centra en el ciclo de modelación elaborado por los autores del presente artículo, teniendo como referencia el ciclo propuesto por Borromeo-Ferri (citado por Huincahue et al., 2018), el cual consta de las siguientes etapas (ver figura 1):

1. Inicia con una situación real que puede ser representada por una imagen, un texto o ambos. En esta etapa se presentan los procesos de experimentación, abstracción, simplificación e interpretación.

2. Se crea una representación mental de la situación para analizar la información de la situación real. 
3. Se produce una transición de teorización del problema para llegar a un modelo real; este proceso es más consciente que el anterior, y dependiendo del problema se involucra el conocimiento extramatemático.

4. Se presenta un proceso de matematización que genera un modelo matemático basado en el conocimiento extramatemático.

5. Se obtienen resultados matemáticos que se interpretan y conducen a resultados reales que se validan en la representación mental o en el modelo real.

Figura 1. Ciclo de modelación matemática propuesto por Werner Blum y Rita Borromeo-Ferri.

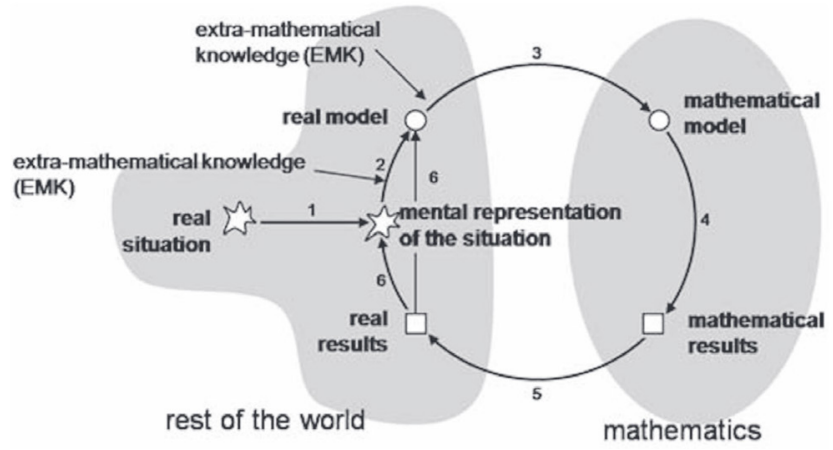

Fuente: Blum y Borromeo-Ferri (2009).

\section{Metodología}

Teniendo en cuenta las fases descritas y el contexto de los estudiantes de Licenciatura en Matemáticas de la Universidad del Atlántico, se ha propuesto un modelo que se ha considerado indicado para la resolución de problemas a partir de la modelación matemática. En la figura 2 se ilustran las fases del proceso de modelación: 
Figura 2. Propuesta de ciclo de modelación matemática.

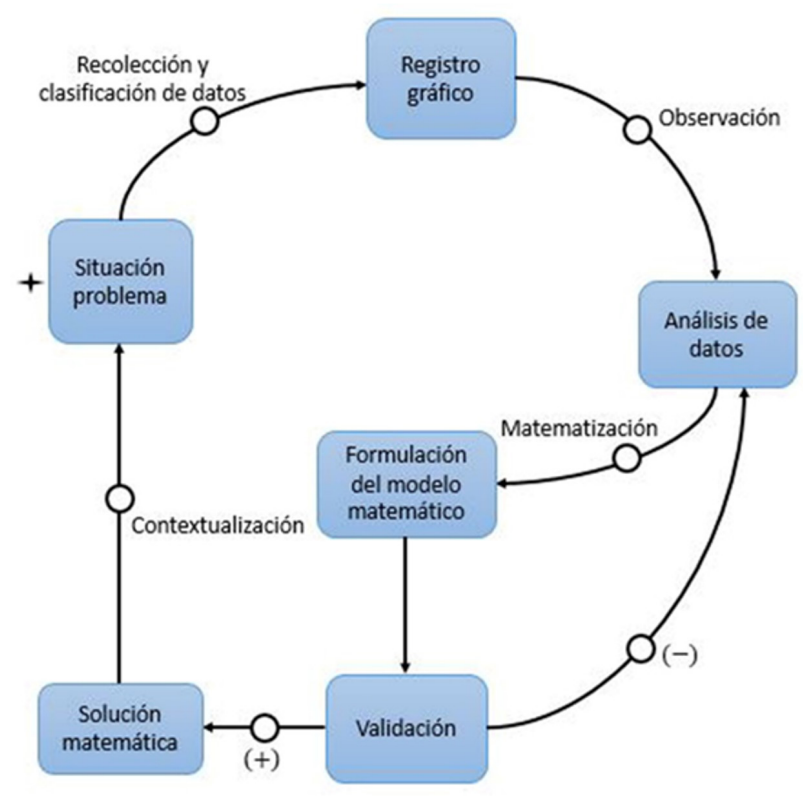

Fuente: elaboración propia.

Este esquema consta de seis fases y entre cada fase se dan procesos intermedios que a continuación se explican:

1. Situación problema: surge de un entorno concreto de la realidad; el cual puede representarse y simularse en distintas formas, ya sean gráficas o mentales, con la finalidad de simplificar y estructurar la situación problema mediante una toma de datos de las variables involucradas en diversos momentos (Cordero et al., 2019). Esto conduce a:

1.1. La recolección de datos y su clasificación, en la cual se espera que el estudiante organice los datos de tal forma que sean lo más entendibles posible. Esto consiste en brindar orden y estructura al proceso de modelación (Villa-Ochoa, Bustamante y Berrío, 2010). 
2. Registro gráfico: en esta etapa se procede a representar los datos de manera gráfica, por ejemplo por medio de diagramas de dispersión, con el fin de facilitar su observación, y así obtener una interpretación geométrica del problema que permita relacionar las distintas variables que intervienen en el fenómeno estudiado. Esta interpretación viene mediada por la:

2.1. Observación: proceso mediante el cual se relacionan los datos obtenidos con una función matemática, la que se considere más conveniente con el fin de conjeturar y formular hipótesis sobre cuál se aproxima mejor a los datos obtenidos.

3. Análisis de datos: una vez ya se ha hecho la observación, se procede a identificar una relación matemática entre las variables que intervienen en el fenómeno estudiado. Con esto se pretende traducir la situación problema, del lenguaje común al lenguaje matemático; es decir:

3.1. La matematización del problema: aquí se espera que el estudiante, una vez tenga identificadas sus variables y la curva que crea que mejor se adapta a ellos, las asocie con constantes y/o parámetros que se deduzcan del análisis previo.

4. Formulación del modelo matemático: a partir del análisis de los datos y su comportamiento, se procede a establecer una ecuación o fórmula matemática que explique la relación previamente identificada. El ideal es construir la mejor aproximación posible al conjunto de datos estudiado a partir de una muestra de estos.

5. Validación del modelo: el objetivo de esta fase consiste en la confrontación de los datos obtenidos con el comportamiento de las variables involucradas y el análisis gráfico que se ha realizado con anterioridad. 
Dependiendo de lo que resulte de esta confrontación se tendrán dos tipos de validación, a saber:

5.1. Validación negativa: los datos obtenidos mediante la fórmula no representan una aproximación a los datos reales o no generan datos con un comportamiento similar a los datos de la experimentación. En este caso de validación negativa, es necesario hacer una nueva revisión de los datos experimentales para corregir o, en su defecto, establecer un nuevo modelo. Esto involucra un análisis más riguroso tanto de los datos como del contexto del experimento.

5.2. Validación positiva: cuando el modelo genera datos con un patrón de comportamiento aproximado a los datos reales, se concluye que la fórmula permite predecir el comportamiento de los datos del fenómeno en un rango de valores más amplio.

6. Solución matemática: si la validación es positiva, se interpreta que la relación resultante de este proceso es el modelo matemático que mejor se aproxima a la solución de la situación problema.

6.1. Contextualización: a partir de lo anterior se procede a delimitar y ubicar dicho resultado en el contexto del problema, es decir, a establecer hasta dónde es válida dicha solución y hasta qué punto se puede reconocer la limitación del modelo, además de establecer predicciones futuras.

\section{Análisis a priori}

El análisis a priori permite poner de manifiesto, antes de la experimentación, relaciones entre las fases expuestas en función de sus características para contrastarlas con el resultado de la experimen- 
tación (Orús, citado por Berrío, 2016). Con el análisis a priori se explicitarán previamente las fases que los licenciados en formación deben seguir a la hora de abordar problemas de modelación matemática.

\section{Análisis a priori de la actividad}

\section{Lanzamiento de dados}

En esta actividad, se lanzaron 100 dados y aquellos que quedaban con la cara superior en 5 se retiraban, el resto se volvía a lanzar y se retiraban nuevamente los dados cuya cara superior era 5, y así sucesivamente hasta completar 10 lanzamientos. Además, se formularon algunas preguntas que los licenciados en formación debían contestar. Los resultados obtenidos se muestran en la siguiente tabla:

Tabla 1. Datos tomados de la experimentación

\begin{tabular}{|c|c|c|c|c|c|c|c|c|c|c|c|}
\hline Número de lanzamientos & 0 & 1 & 2 & 3 & 4 & 5 & 6 & 7 & 8 & 9 & 10 \\
\hline Cantidad de dados restantes & 100 & 77 & 73 & 61 & 51 & 45 & 37 & 31 & 25 & 20 & 14 \\
\hline
\end{tabular}

Fuente: elaboración propia.

\section{Preguntas}

1. ¿Qué variables identificas en la experiencia?

2. ¿Cómo se comportan los datos?

3. ¿Qué relación puede establecerse entre las variables?

4. ¿Qué tipo de función representa la experiencia?

5. Para los lanzamientos número 15 y 16 ¿cuántos dados quedarán?

6. ¿En qué tiempo ya no quedarán dados?

\section{Objetivo}

Analizar los distintos pasos o secuencias que siguen los licenciados en formación para poder establecer el modelo matemático que 
represente la situación problema, de tal manera que pueda esquematizarse dicha secuencia con el fin de contrastarla con el ciclo de modelación propuesto anteriormente.

\section{Descripción}

Teniendo en cuenta el ciclo de modelación propuesto, se espera que los licenciados en formación, al realizar el experimento, puedan identificar las variables que intervienen en este. Para esto deberán recolectar y clasificar los datos de tal manera que puedan sistematizarlos, es decir, organizarlos y clasificarlos para su posterior representación gráfica (un diagrama de dispersión).

Con esto, se pretende que mediante la dispersión de los datos los licenciados en formación realicen las respectivas observaciones sobre ellos, con la finalidad de comparar o conjeturar qué funciones o relaciones conocidas se ajustan mejor al diagrama de dispersión.

Dependiendo de lo que los licenciados en formación puedan inferir de las observaciones anteriores, se espera que procedan a establecer una relación matemática entre las variables que intervienen en la experiencia, es decir, la población (cantidad de dados) y el tiempo (número de lanzamientos).

Esto los llevará a la matematización de la experiencia, para esto y a partir de las variables definidas con anterioridad y con base en la dispersión, el estudiante notará que su modelo es exponencial, como se muestra en la figura 3. 
Figura 3. Diagrama de dispersión de los datos de la tabla 1.

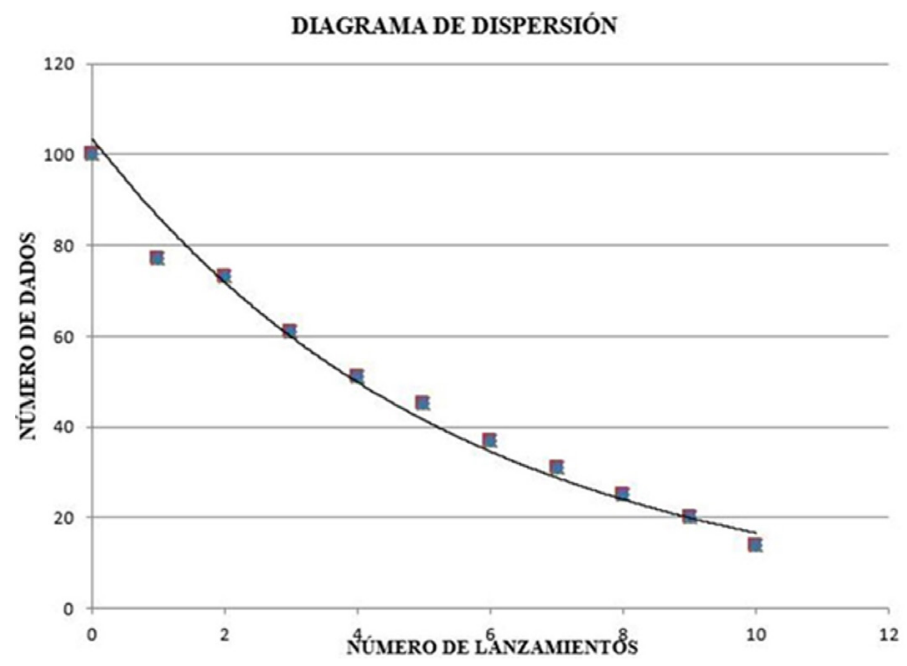

Fuente: elaboración propia.

Con base en el comportamiento de los datos, identificará que se trata de un problema de decaimiento poblacional. Una vez haya podido realizar esto, procederá a la matematización del problema, es decir, a determinar que la variación de la población ( $d p)$ respecto al tiempo $(d t)$ es directamente proporcional a la población en ese tiempo $(p(t))$. Tal y como se muestra a continuación.

$$
\frac{d p}{d t} \propto p(t)
$$

Lo anterior nos lleva a que

$$
\frac{d p}{d t}=k \cdot p(t)
$$

Esta es una ecuación diferencial de variables separables, la cual nos conduce a la formulación del modelo matemático, es decir, una fórmula matemática que explique la relación previamente identificada, que para este caso vendría siendo la solución de la ecuación diferencial anterior, y sería la siguiente: 


$$
p(t)=c \cdot e^{k \cdot t}
$$

Una vez se ha formulado el modelo formalmente, se espera que los licenciados en formación procedan a realizar la validación o verificación de este, a partir de procedimientos de ensayo y error, es decir, darles valores a las variables utilizando los datos de la tabla para saber qué tan aproximado es el modelo.

Esta verificación, dependiendo de las aproximaciones obtenidas, puede llevar a dos caminos:

- Validación negativa: si los datos obtenidos mediante el empleo de la fórmula de decrecimiento poblacional no son aproximados a los datos reales obtenidos en el experimento, se espera que el licenciado en formación realice una verificación de los datos experimentales para corregir o, en su defecto, proporcionar un nuevo modelo matemático. Esto debe llevarlos a un análisis más riguroso de los datos y el contexto.

- Validación positiva: si los datos obtenidos generan una buena aproximación a los datos reales, se espera que los licenciados en formación utilicen dicho modelo para establecer predicciones fuera del rango de datos obtenidos inicialmente (responder la pregunta 5).

Si de lo anterior el licenciado obtiene que su validación es positiva, deberá proponer ese modelo matemático como la solución del problema junto con los datos obtenidos, los cuales a su vez se deberán delimitar y ubicar en el contexto del problema, y analizar hasta dónde es válida dicha solución y hasta qué punto se puede reconocer la limitación del modelo (responder la pregunta 6). Además de esto, los licenciados en formación podrán formular hipótesis respecto al modelo planteado y su aplicación en otras áreas del conocimiento. 


\section{Resultados}

\section{Análisis a posteriori}

Este análisis tiene como objetivo explicitar los procesos de razonamiento detectados, las estrategias de solución propuestas y las acciones realizadas en cada etapa en comparación con los datos previstos en el análisis a priori (Berrío, 2016).

\section{Análisis a posteriori de la actividad}

Al comenzar la actividad se planteó la dinámica de la experiencia y algunos interrogantes que debieron resolverse posteriormente. Primeramente, se lanzaron los dados, y luego los licenciados en formación registraron los datos en su cuaderno de notas o apuntes, como lo muestra la figura 4.

Figura 4. Registro de datos.

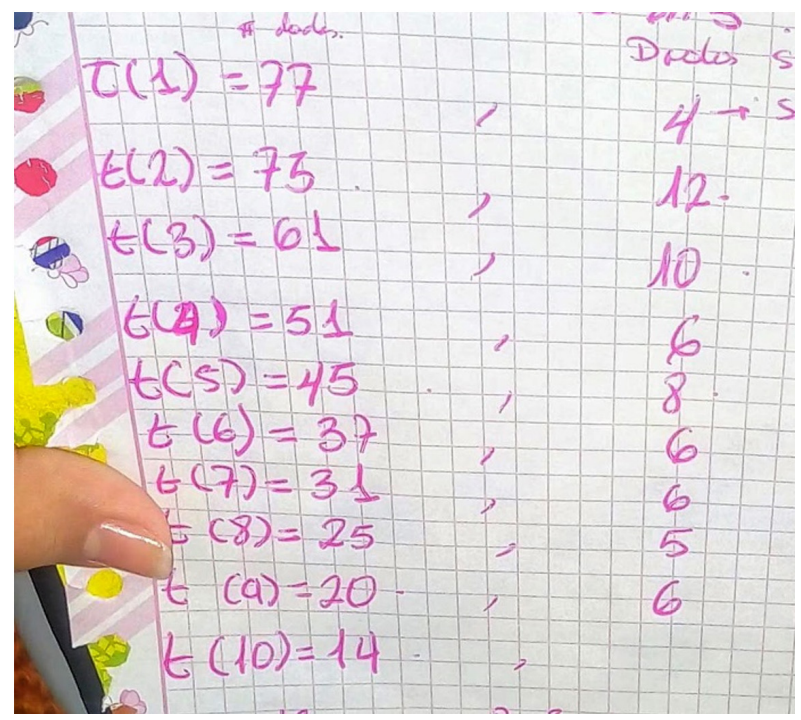

Fuente: elaborado por estudiantes. 
Al terminar la experiencia con los dados, los licenciados en formación procedieron a realizar una organización de los datos obtenidos en tablas en la medida en que se iban obteniendo, como se muestra en la figura 5 .

Figura 5. Tabulación de datos.

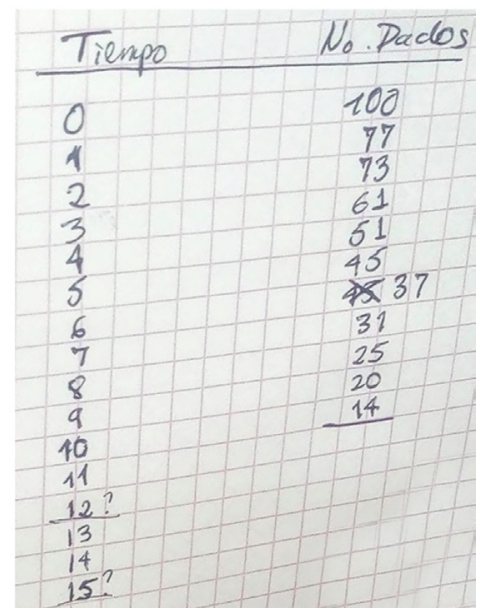

Fuente: elaborado por estudiantes.

Una vez hicieron esto, procedieron a realizar la matematización de los datos, es decir, establecer una relación entre las variables que identificaron mediante procesos aritméticos, como la diferencia entre el número total de dados y los dados que se iban retirando en cada lanzamiento (figura 6).

Figura 6. Matematización de los datos

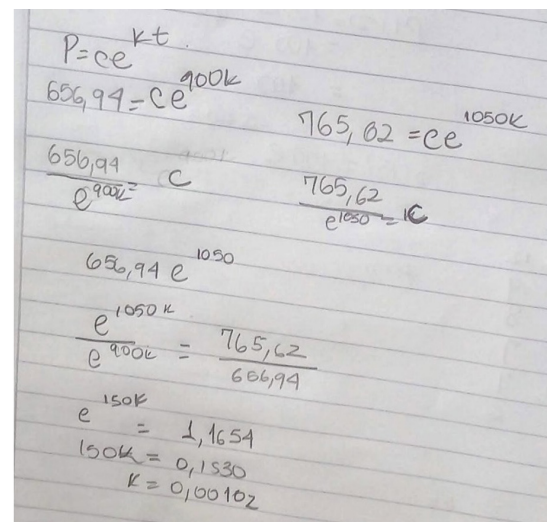

Fuente: elaborado por estudiantes. 
Otros, sin hacer un análisis completo del comportamiento de los datos, y con base en las inferencias hechas a partir de estos, plantearon el modelo matemático de decaimiento poblacional. Aquí no se hizo la matematización del problema, sino que formularon el modelo debido a que ya habían tomado un curso de ecuaciones diferenciales.

Luego de esto empezaron a hacer pruebas con el modelo, es decir, verificaciones utilizando algunos datos de la tabla, como el primero y el segundo, lo que produjo que el modelo que plantearon no fuese satisfactorio para el fenómeno estudiado; por lo tanto, los licenciados en formación cambiaron los datos que tomaron para encontrar los valores de las constantes c y $\mathrm{k}$ que intervienen en el modelo, y nuevamente verificaron si era una aproximación más cercana que la que habían encontrado anteriormente. Aquellos que consideraron que su aproximación era correcta procedieron a resolver los interrogantes planteados.

\section{Análisis de resultados}

Partiendo de lo anterior, observamos que los licenciados en formación, si bien organizaron los datos que se iban obteniendo en tablas, no hicieron una representación gráfica de estos, sino que de inmediato procedieron a matematizar la situación planteada, dejando de lado el registro gráfico, lo que influyó en su posterior interpretación y análisis, pues obviaron un aspecto importante que se propone en el ciclo de modelación, como lo es la observación.

Los licenciados en formación, al plantear la fórmula que según ellos modela la experiencia realizada en el aula, mediante datos tomados de la tabla, se dieron cuenta al realizar la validación de que los valores que arrojaba el modelo propuesto no eran aproximados a los datos obtenidos inicialmente. Ante esto, en vez de retroceder a los datos nuevamente, hacer un registro gráfico y analizar el comportamiento del fenómeno como se enunció en el análisis a priori, simplemente cambiaron los datos que utilizaron para la fórmula planteada al inicio, lo que no va en concordancia con el ciclo 
propuesto en este artículo, el cual prevé que en dado caso que la validación sea negativa se debe hacer nuevamente un análisis riguroso de los datos, lo que debe generar un cambio en el modelo propuesto, o en su defecto un nuevo modelo matemático.

Por último, los resultados que se obtuvieron de dichos modelos no fueron contextualizados en la situación problema para resolver los interrogantes planteados, es decir, sus conclusiones a predicciones futuras estaban fuera de la realidad, teniendo en cuenta que el cero es una asíntota de la función exponencial; por consiguiente, para valores muy grandes de $t$ la cantidad de dados nunca iba a ser cero.

Con base en lo anterior y teniendo en cuenta lo que los licenciados en formación hicieron a lo largo del experimento, se esquematizaron los pasos o las fases que iban mostrando al abordar la situación problema. Tales pasos se representan en la figura 7 .

Figura 7. Esquema del proceso de modelación desarrollado por los estudiantes.

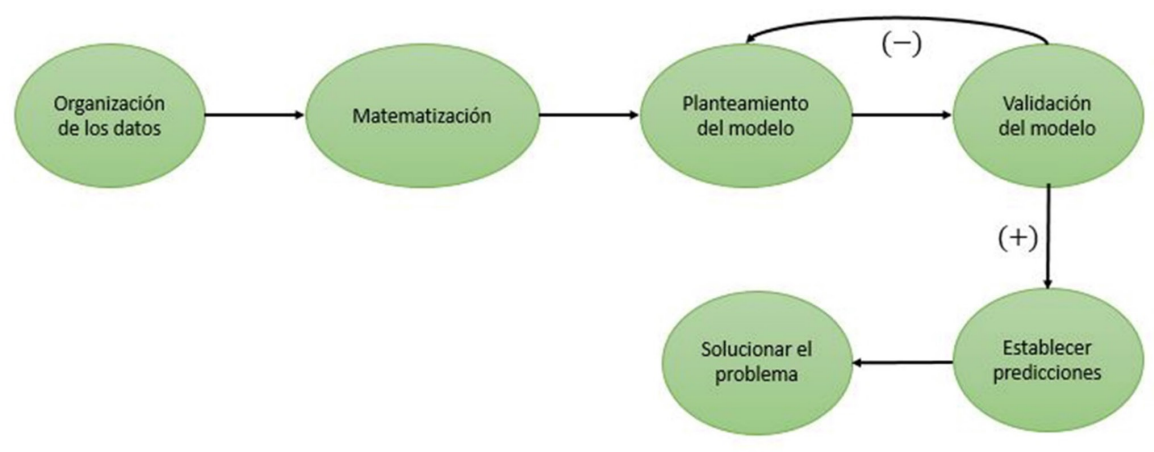

Fuente: elaboración propia.

\section{Contraste}

Con base en el ciclo propuesto en este artículo, el análisis a priori y el esquema que sintetiza el desarrollo de la modelación matemática por parte de los licenciados en formación, se presenta el siguiente contraste: 
- En la figura 2, el ciclo de modelación inicia con una situación problema de la realidad, la cual se les planteó a los licenciados en formación, que fueron obteniendo sus datos a partir de la experimentación y su participación directa en ella. En la figura 7 se evidencia que los licenciados en formación no tuvieron en cuenta este aspecto, aunque de manera inconsciente participaron de él.

- Como se esperaba, los licenciados en formación recolectaron y clasificaron sus datos según la figura 2, pero procedieron directamente a la matematización del problema, dejando por fuera el registro gráfico y por consiguiente el proceso de observación propuesto, lo que limita el análisis e interpretación del fenómeno estudiado y tiene consecuencias que se hicieron notables al momento del planteamiento del modelo, fase a la que procedieron después de la matematización, como se ilustra en la figura 7.

- Al intentar validar el modelo, que formularon dado que ya habían visto un curso de ecuaciones diferenciales y sabían la fórmula, muchos no eligieron bien sus datos, lo que los llevó a soluciones muy lejanas incluso a los datos obtenidos en la tabla 1. Así, procedieron simplemente a cambiar estos datos por otros, sin tener en cuenta lo propuesto en la figura 2 respecto a la validación negativa.

- Aquellos cuya validación fue positiva, si bien contestaron satisfactoriamente la pregunta 5 y establecieron las respectivas predicciones como lo ilustra la figura 7, fueron incapaces de resolver la pregunta 6, pues no supieron contextualizar dicho resultado en el problema planteado, tal y como señala la figura 2; por el contrario, procedieron a dar la solución del problema (la respuesta a la pregunta 5) y a determinar como irresoluble la pregunta 6. 


\section{Conclusiones}

En el curso de Didáctica del Cálculo de la Universidad del Atlántico se les planteó a licenciados en formación la actividad descrita en este artículo. A partir de los resultados obtenidos y su análisis, se concluye lo siguiente:

- Existen dificultades en la resolución de problemas de modelación matemática por parte de licenciados en matemáticas en formación, pues la noción de modelación matemática que tienen consiste en una aplicación o problema matemático y esto se debe a que frecuentemente, los docentes presentan las situaciones problemas a manera de formulación, limitando así la complejidad del mismo, además de restarle importancia al proceso de validación (Cuenca, Palauro, Astiz y Vivera, 2019) lo que conlleva que en la escuela estas prácticas y razonamientos no se presenten adecuadamente.

- Es necesario presentar la modelación matemática en un escenario de prueba y experimentación para que los estudiantes desarrollen los distintos procesos que en ella intervienen y de esta manera puedan articular las matemáticas con otras áreas del conocimiento, pues la forma en cómo se produce el aprendizaje significativo está en relación con los contextos y el entorno en que estos se pretendan enseñar (Márquez, Gaviria y López, 2019).

- Se evidencia una fuerte aceptación por parte de los estudiantes a las actividades de experimentación en el aula, pues estas les permiten tener una mejor contextualización de los problemas de modelación matemática. Lo que para Villa-Ochoa, Bustamante, Berrío, Osorio y Ocampo (2009) es importante pues, las concepciones de realidad que tienen los profesores marcan ciertas directrices para abordar los procesos de modelación en las aulas de clase. 
- El realizar actividades de experimentación en el aula, les da a los licenciados en formación una noción de realidad más concreta, lo que permite realizar inferencias ajenas a los marcos abstractos de la solución, teniendo así respuestas incongruentes con la realidad y entendiendo que el modelo tiene sus limitaciones al tratarse de una aproximación al fenómeno estudiado.

- Las actividades de experimentación en el aula benefician el sentido de realidad que los estudiantes tienen de los fenómenos que a menudo se presentan en enunciados; estas actividades brindan un valor más concreto de los datos que se obtienen en estudios más globales.

\section{Referencias bibliográficas}

Berrío, J. D. (2016). Estudio de la construcción de pasos de razonamiento en el proceso de justificación teórica en la resolución de problemas de geometría (tesis de maestría). Universidad Industrial de Santander, Bucaramanga, Colombia.

Berrío, J., Peña, Z. y Torrenegra, M. (2018). Estrategias didácticas para el desarrollo de procesos de modelación con ecuaciones diferenciales desde la perspectiva STEM. Memorias: Cuarto Encuentro Internacional de Investigación en Educación Matemática (pp. 347-352). Puerto Colombia, Universidad del Atlantico.

Biembengut, M. y Hein, N. (1999). Modelación matemática: estrategia para enseñar y aprender matemáticas. Educación Matemática, 11(1), 119-134.

Biembengut, M. y Hein, N. (2004). Modelación matemática y los desafíos para enseñar matemática. Educación Matemática, 16(2), 105-125. 
Blum, W., Galbraith, P. L., Henn, H.-W. y Niss, M. (eds.). (2007). Modelling and Applications in Mathematics Education: The 14th ICMI Study. Nueva York: Springer Science+Business Media.

Blum, W. y Borromeo-Ferri, R. (2009). Mathematical Modelling: Can it Be Taught and Learnt? Journal of Mathematical Modelling and Application, 1(1), 45-58.

Cordero, F., Villa-Ochoa, J., Rosa, M., Suárez-Téllez, L., Carranza, P. y Mendoza-Higuera, E. (2019). La modelación en la matemática educativa: sus métodos de investigación y el impacto educativo en la formación y desarrollo de la docencia de la matemática. Acta Latinoamericana de Matemática Educativa, 32(1), 549-557.

Cuenca, M., Palauro, L., Astiz, M. y Vivera, C. (2019). La modelización matemática: análisis de entrevistas a docentes y su material de clases. Revista de Educación, 16, 161-172.

Fernández-Sánchez, O. y Angulo-Cruz, M. (2019). Proceso de modelación en clase de matemáticas. Scientia et Technica, 24(1), 97-103.

Huincahue, J., Borromeo-Ferri, R. y Mena-Lorca, J. (2018). El conocimiento de la modelación matemática desde la reflexión en la formación inicial de profesores de matemática. Enseñanza de las Ciencias, 36(1), 99-115.

Márquez, C., Gaviria, C. y López, Y. (2019). Evaluación del desarrollo de competencias a partir de la modelación matemática. Ingenierías USBMed, 10(2), 8-15.

Ministerio de Educación Nacional. (1998). Lineamientos curriculares: matemáticas. Bogotá: Magisterio.

Morales, J. y Currea, J. (2019). Un ejercicio de modelación matemática, aplicando los conceptos del álgebra lineal con estudiantes de la Facultad de Ingeniería y el programa de Economía de la Universidad Católica de Colombia. Encuentro de Ciencias Básicas, 2, 15-25. 
Ortega, M., Zamora, D., Ulloa, J. y González, J. (2018). La modelación en el aprendizaje de las matemáticas. Revista EducateConCiencia, 18(19), 65-78.

Pantoja, R., Guerrero, M., Ulloa, R. y Valdivia, S. (2016). La modelación matemática en situaciones problema de la vida cotidiana. Artículo presentado en el Seminario Repensar las Matemáticas, sesión 83. Recuperado de https://repensarlasmatematicas.files.wordpress. com/2016/03/s83-documento-de-referencia-bis.pdf

Rodríguez, R. (2010). Aprendizaje y enseñanza de la modelación: el caso de las ecuaciones diferenciales. Revista Latinoamericana de Investigación en Matemática Educativa, Relime, 13(4), 191-210.

Rodríguez, R. y Quiroz, S. (2016). El rol de la experimentación en la modelación matemática. Educación Matemática, 28(3), 91-110.

Suárez, L. y Cordero, F. (2010). Modelación-graficación, una categoría para la matemática escolar: resultados de un estudio socioepistemológico. Revista Latinoamericana de Investigación en Matemática Educativa, Relime, 13(4-II), 319-333.

Trigueros, M. (2009). El uso de la modelación en la enseñanza de las matemáticas. Innovación Educativa, 9(46), 75-87.

Villa-Ochoa, J. y Ruiz, H. (2009). Modelación en educación matemática: una mirada desde los lineamientos y estándares curriculares colombianos. Revista Virtual Universidad Católica del Norte, 27, 1-21.

Villa-Ochoa, J., Bustamante, C. y Berrío, M. (2010). El pensamiento del docente, sus prácticas y elementos para su formación profesional. En Lestón, P. (ed.), Acta Latinoamericana de Matemática Educativa, 23 (pp. 1087-1096). Ciudad de México: Comité Latinoamericano de Matemática Educativa A. C.

Villa-Ochoa, J., Bustamante, C., Berrío, M., Osorio, J. y Ocampo, D. (2009). Sentido de realidad y modelación matemática: el caso de Alberto. Alexandria, 2(2), 159-180. 